\title{
Impact of Dust and Sand on 5G Communications for Connected Vehicles Applications
}

This paper was downloaded from TechRxiv (https://www.techrxiv.org).

\section{LICENSE}

CC BY 4.0

SUBMISSION DATE / POSTED DATE

$16-01-2022$ / 20-01-2022

\section{CITATION}

Abuhdima, Esmail (2022): Impact of Dust and Sand on 5G Communications for Connected Vehicles Applications. TechRxiv. Preprint. https://doi.org/10.36227/techrxiv.18482000.v1

$\mathrm{DOI}$

10.36227/techrxiv.18482000.v1 


\title{
Impact of Dust and Sand on 5G Communications for Connected Vehicles Applications
}

\author{
Esmail Abuhdima, Member, IEEE, Jian Liu, Chunheng Zhao, Ahmed Elqaouaq, Gurcan Comert, \\ Chin-Tser Huang, Senior Member, IEEE, Pierluigi Pisu, Member, IEEE, Amir hossein Nazeri
}

\begin{abstract}
Recent research activities focused on improving Vehicle-toVehicle Communication (V2V) based on the 5G Technology. V2V applications are important because they are expected to reduce the risk of accidents up to $80 \%$, enhance traffic management, mitigate congestion, and optimize fuel consumption. Typical autonomous vehicle applications require a high bandwidth transmission channel, so the $5 \mathrm{G}$ communication channel is a reliable solution to support this technology. The dedicated shortrange communications (DSRC), characterized by a frequency bandwidth of $5.9 \mathrm{GHz}$, were used as vehicular connectivity with bandwidth up to $200 \mathrm{mb} / \mathrm{s}$ and limited capacity, and it is here utilized for comparison to 5G. The 5G band can support connected autonomous vehicles with high data rates and large bandwidth. The 5G communication channel is considered for vehicular connectivity since it has a very high bandwidth in the millimeter waves spectrum range. The quality of $5 \mathrm{G}$ wireless communication channels between connected vehicles is affected by weather conditions such as rain, snow, fog, dust, and sand. In this paper, the effect of dust and sand on the propagation of millimeter waves is presented. The effect of dust and sand on the communication path loss of DSRC and 5G frequency band is investigated in the case of Urban areas and the Highway condition. Results show that the attenuation caused by dust and sand depends on the particle size of sand, frequency of propagating wave, and concentration of dust. Finally, a new model of link margin is proposed to estimate the effect of dust and sand on DSRC (5.9 GHz) and $5 \mathrm{G}(28 \mathrm{GHz}-73.5 \mathrm{GHz})$ communication path loss.
\end{abstract}

Index Terms-Vehicle-to-vehicle communications, 5G, weather, path loss, traffic intersection, and millimeter waves.

\section{INTRODUCTION}

$\mathbf{T}$ HIS paper is an expansion of the previous study published in 2021 IEEE International Conference on Wireless for Space and Extreme Environments (WiSEE) [1]. Intelligent transportation systems (ITS) contain the most upcoming technologies that span different traffic applications involving vehicle-to-vehicle $(\mathrm{V} 2 \mathrm{~V})$, vehicle-to-infrastructure (V2I), vehicle-to-pedestrian (V2P), and vehicle-to-network (V2N) communications. They help to increase the efficiency of traffic, reduce crashes, ease traffic congestion, and improve fuel efficiency [2], [3]. However, many ITS applications require reliable communication to support integral functions such as intersection control, queue warning, gap assistance, curve

This paper was funded by U.S. DOT Tier 1 University Transportation Center, the Center for Connected Multimodal Mobility $\left(C^{2} M^{2}\right)$. Esmail Abuhdima, Gurcan Comert, and Ahmed Elqaouaq are with Benedict College, Columbia, SC 29204 USA. Jian Liu and Chin-Tser Huang are with University of South Carolina, Columbia, SC 29208 USA. Chunheng Zhao, Pierluigi Pisu, and Amirhossein Nazeri are with Clemson University, Clemson, SC 29634 USA

Manuscript received January 15, 2022; revised. speed warning, and alike. Disruption on the communication would result in catastrophic results such as collisions and costly congestion [4]. Since 5G can support connected autonomous vehicles with a high data rate, the $5 \mathrm{G}$ wireless communication is considered one of the connected vehicles solutions. De facto, $5 \mathrm{G}$ wireless communication is becoming the optimum communication channel used to support new features. The quality of wireless communication channels between vehicles at high frequencies can be potentially affected by weather conditions such as rain, snow, fog, dust, and sand. Due to the absorption of the energy of the transmitted signal and the scattering at high frequencies, the dust and sand cause significant attenuation at these high-frequency bands, especially at the E-band. The effect of the dust and sand on the propagating signal can show up for four different reasons: (i) the backscattered signal, (ii) the interference according to the time delay of the received signal, (iii) change the polarization of the transmitted signal, and (iv) absorption of the energy of the transmitted signal. Climate circumstances influence wireless communication channels. Usually, in the northern and southeastern U.S. regions, these communication channels are affected by climatic factors like rain, snow, and humidity. In contrast, these channels are affected more by dust and sand storms in the dry southwest regions. Such climate factors absorb and scatter part of the information of the transmitting signals. The performance of service of many wireless applications, such as 5G networks, cellular telephones, public service radio, pagers, broadcast television, radio stations, and differential GPS transmitters, CDMA and WiMax networks, Wifi and Bluetooth networks that require RF or microwave propagation from point to point very near the Earth's surface, depends on many factors such as area of coverage and climate conditions. When the propagating electromagnetic waves pass through a medium containing precipitations like sand and dust particles, the signals are attenuated through absorption and scattering of the energy by particles of the sand and dust [5]. It is seen that the Vehicle-to-Vehicle (V2V) communications have received increasing attention, but it is required to conduct deep analysis of certain important quantities such as path loss.

In the literature, empirical path loss models were developed using measurement data for different environmental areas [6][8]. These studies considered the effect of distances between vehicles, small and large-vehicle obstruction, climate factors such as rain and fog, and the height and characteristic of the antenna on the path loss. Also, the effect of sand and dust storms on wireless communication, such as microwave links and GSM signal coverage was addressed, and was found that the effect of sand and dust attenuates the propagating signal 
[5]. This paper addresses path loss analysis and modeling for Vehicle-to-Vehicle communications with sand and dust storms by determining the attenuation factors. The attenuation and phase shift constants for a medium with dust or/and sand particles depend on the frequency, visibility, greatest particles size, shape of the scattering particles, concentration, and orientation relative to the wave polarization.

This paper discusses the weather effect on $5 \mathrm{G}$ communications, particularly the impact of dust and sands on the propagating $\mathrm{mm}$-Wave [1]. The difference between the dust and sand particles is the diameter, where the sand diameter is larger than the dust particles' diameter. The dust particle size is ranged from $10 \mu \mathrm{m}$ to $80 \mu \mathrm{m}$, and the sand particle size is ranged from $0.15 \mathrm{~mm}$ to $0.3 \mathrm{~mm}$. The attenuation caused by the dust and sand is one of the major problems in the use of terrestrial and space wireless communication (microwave and mm-wave signals). One misconnection can cause significant damage to circuit traffic, and this study aims to help provide a better reliable connection between the vehicles in this weather condition. There are limited studies in the literature on the impact of weather on 5G ( $\mathrm{mm}$-Wave) communications [9]. Only a handful of them discusses the effect on vehicular communications. Previous research studied attenuation and backscattering effects were incorporated via formulations and quantified the weather particle sizes impacts [2] using Monte Carlo simulations. In general, studies focused on the impact on the communication range. According to these studies, a range of $1.8 \mathrm{~km}$ would drop to $300 \mathrm{~m}$ with heavy rain and fog while snow would induce noise in communications during the attenuation of the received signal. Hence, applications such as intersection control with such reductions would have increased risks. For instance, in an isolated intersection, we would expect to track up to at least $200 \mathrm{~m}$ (e.g., $150 \mathrm{~m}$ was given in [10]).

The empirical path loss models were developed using measurement data for different environmental areas [2], [6], [9]. These results considered the effect of distance between vehicles, small and large-vehicle obstructions, climate factors such as rain and fog, and the height and characteristic of the antenna on the path loss. Also, the effect of sand and dust storms on wireless communication, such as microwave links and GSM signal coverage was addressed and it was found that the propagating signal is attenuated by the effect of sand and dust [5], [11]. In this paper, 5G mm-wave is proposed as a solution to the connectivity of the vehicle since the $5 \mathrm{G}$ can support multiple connected autonomous vehicles with high data rate and massive transmitting bandwidth. For these main reasons, the $5 \mathrm{G}$ wireless communication channel is considered as the vehicular connectivity preferable option. This paper considers most of the wireless interactions between the selfdriving vehicles on a low-height level, approximately a range between $1 \mathrm{~m}$ to $4 \mathrm{~m}$.

Currently, there are alternative $5 \mathrm{G}$ mm-Wave simulators available. Some of these simulators were investigated for this study. The first simulator considered was NYUSIM [12]. NYUSIM is an open-source mm-Wave channel simulator and is written in MATLAB. It has an interface to change channel parameters, antenna properties and so on. The simulator's functions are all predefined. The second simulator was the $5 \mathrm{G}$
Toolbox [13] in MATLAB. This toolbox supports link-level simulation and test waveform generation. It has a waveform generator and transmitter. Simu5G [14] is another open source simulator based on OMNeT++ framework, it allows one to simulate network scenarios where $4 \mathrm{G}$ and $5 \mathrm{G}$ coexist, in both StandAlone (SA) and E-UTRA/NR Dual Connectivity (ENDC) deployments. Millicar, a mmWave-based V2X network simulator based on NS-3, has the functionality of simulating vehicular networks with a 3GPP channel model [15]. Among these simulators, due to functionality of direct modification of the path loss function and smoother interaction with vehicle mobility, we utilized the NS-3-based Millicar simulator to study the weather impact on the $5 \mathrm{G}$ communication channel for connect vehicles [15].

In this paper, the effect of dust and sand with standards of the visibility, particle size, and different humidity levels $(0 \%$, $60 \%$, and $100 \%$ ) on the $5 \mathrm{G}$ vehicle channel is investigated in comparison with the dedicated short-range communication channel, DSRC, $(5.9 \mathrm{GHz})$. Two possible scenarios are considered to estimate the path loss of the propagating signal. These scenarios are Urban and Highway conditions. These scenarios are according to the main driving categories, but they have different conditions. The path loss for each condition should be calculated differently to estimate the accurate value of the link margin. The specific formula that is used for each condition to estimate the path loss will be shown in Section III. Along with transmission parameters of used sensors such as transmitted power, antenna gain, and total free space loss; the attenuation of the transmitted signal is computed in terms of operating frequency, the concentration of dust, and particle size of sand. According to the received signal, it can determine whether the attenuation of the signal is high or low in comparison with the threshold of the received power. The worst weather condition is considered to calculate the attenuation factor in $d B / \mathrm{km}$, when the visibility is low and the particle size of dust and sand is large.

This paper is organized into five main sections. Section II will generally discuss the main topic of the research that studied the effect of dust and sand storms on the propagating $5 \mathrm{G}$ mm-wave. Section III describes the effect of dust and sand on the Link Budget of the communication channel. The region of study and research measurements are also mentioned in Section IV. The numerical experiments and results are discussed in Section V. Finally, the conclusion and recommendations are represented in Section VI.

\section{SAnd And Dust Attenuation}

\section{A. Definition of dust and sand}

In general, attenuation is the loss of the transmitting signal strength in the transmission media. This transmission media could be wireless channel, cables or satellite link. In this study, the path loss of the wireless channel $r$ is derived in terms of frequency, propagating path, visibility, and permittivity of transmission media. The deserts across the planet are a resource of the dust and sand particles that are distributed globally, and those deserts make up approximately $20 \%$ of the Earth's surface. For example, the desert of North Africa 
is the source of dust that affects Southern Europe [16]. Also, the desert of the southwest of the United States is the source of dust and sand in this country [16]. The proposed dust and sand attenuation model can be used to determine the effect of dust and sand storms on the propagating $\mathrm{mm}$ wave when the concentration of the dust and the radius of particle size vary according to the change of the weather condition. This attenuation factor is dependent on different variables or parameters, such as operating frequency, humidity, height, particle size, and visibility. These factors contribute to a variation of the attenuation factor, so if these parameters change, the attenuation factor will vary as well. Frequency is the number of occurrences that a wave surpasses a point within a specific amount of time. Frequency is measured in the unit of Hertz $(\mathrm{Hz})$. Humidity is the measure of how much water vapor is in the air. Height is a measure of distance above the ground where the attenuation factor is computed. Visibility is the measure of distance that a human being can see an object during dust and sand storms. All of these factors are considered when the attenuation factor is investigated. The visibility at reference height $h_{0}$ in $m$ and reference visibility $V_{0}$ in $\mathrm{km}$ is defined in [17] and presented in (1)

$$
V^{\gamma}=V_{0}^{\gamma}\left[\frac{h}{h_{0}}\right]^{b}
$$

where $\gamma$ is a constant that depends on the distance from the point of origin of the storm type of soil and climatic conditions at the origin, $\mathrm{b}$ is a constant that depends on the climatic conditions, meteorological factors, and the particle size distribution of the dust and the sand, and $h$ is the height $m$ from the ground [18].

The important direct measurement to investigate the effect of dust and sand storms is to predict the dielectric constant for dust and sand particles from their mineral and/or chemical composition values. The complex permittivity of the composite component $\epsilon_{m}$ is computed using the Looyenga Equation (2) as given by [19]

$$
\epsilon_{m}^{1 / 3}=\sum_{i=1}^{n} v_{i} \epsilon_{i}^{1 / 3}
$$

where $\epsilon_{i}$ is the complex dielectric constant of the $i^{t h}$ substance and $v_{i}$ is the relative volume of the $i^{t h}$ sample from the volume of the total sample. The permittivity of transmission media is written as $\epsilon=\epsilon_{1}-\epsilon_{2}$, where $\epsilon_{1}$ is the dielectric constant and $\epsilon_{2}$ is the dielectric loss factor.

\section{B. Attenuation Model}

The mathematical model, which is based on the Mie scattering method, is developed to compute the reduction of the propagating mm-wave strength. The ratio of diameter (sand/dust) to the wavelength of the propagating signal is considered when the attenuation factor model is developed to get an accurate effect. This model is valid to use, especially at higher frequencies. The most important parameters that affect the attenuation value are particle radius, operating frequency, humidity, and complex permittivity. The attenuation of the dust and sand $A_{d}$ is defined by [20], [21] in $d B / \mathrm{km}$ and presented in (3)

$$
\begin{gathered}
A_{d}=\frac{a_{e} f}{v}\left[C_{1}+C_{2} a_{e}^{2} f^{2}+C_{3} a_{e}^{3} f^{3}\right] \\
C_{1}=\frac{6 \epsilon_{2}}{\left(\epsilon_{1}+2\right)^{2}+\epsilon_{2}^{2}} \\
C_{2}=\epsilon_{2}\left[\frac{6\left[7 \epsilon_{1}^{2}+7 \epsilon_{2}^{2}+4 \epsilon_{1}-20\right]}{\left[\left(\epsilon_{1}+2\right)^{2}+\epsilon_{2}^{2}\right]^{2}}+\frac{1}{15}+\frac{5}{3\left[\left(2 \epsilon_{1}+3\right)^{2}+4 \epsilon_{2}^{2}\right]}\right] \\
C_{3}=\frac{4}{3}\left[\frac{\left(\epsilon_{1}-1\right)^{2}\left(\epsilon_{2}+2\right)+\left[2\left(\epsilon_{1}-1\right)\left(\epsilon_{1}+2\right)-9\right]+\epsilon_{2}^{4}}{\left[\left(\epsilon_{1}+2\right)^{2}+\epsilon_{2}^{2}\right]^{2}}\right]
\end{gathered}
$$

where $a_{e}$ is the equivalent particle radius in meters, $v$ is the visibility in $k m, f$ is the frequency in $\mathrm{GHz}, C=2.3 \times 10^{-5}$, $\gamma=g=1.07$, and $b=0.28$ [13]. The attenuation of dust and sand in $d B$ is defined as [1]

$$
\alpha_{(d B)}=\int_{0}^{d} A_{d} d l
$$

where $d$ is the length of propagation wave.

\section{Link BUdGet ANALYSIS With DUST AND SAND}

First of all, the path loss is the loss or attenuation on the power of a propagating wave as it propagates through transmission media. In this study, the free space is considered as a transmission media. According to (3), when the dust and sand particles in the air increase, the path loss is increases too. The importance of path loss is that it is needed to find the total received power of signals being transmitted from point to point, such as vehicle to vehicle or vehicle to infrastructure. In the case of $\mathrm{V} 2 \mathrm{~V}$ connection, there are two possible scenarios that are studied for path loss. These scenarios are Urban condition and Highway condition. These two scenarios were specifically chosen because they are general driving environments regardless of the general locations. The path loss for each case should be calculated differently to derive an accurate value of signal' loss. The path loss of the Urban condition is defined as [6]

$$
L_{s}=38.77+16.7 \log _{10}(d)+18.2 \log _{10}(f)+\chi_{a}
$$

The path loss of the Highway condition can be written as

$$
L_{s}=23.4+20 \log _{10}(d)+20 \log _{10}(f)+\chi_{a}
$$

where $f$ is the signal frequency in $\mathrm{GHz}, d$ is the distance between vehicles in meters and $\chi_{a}$ represents the shadowing. According to dust and sand storm conditions, the expression of the path loss for both conditions will be modified to take into account the effect of dust and sand. The modified path loss of Urban condition is

$$
L_{s m}=38.77+16.7 \log _{10}(d)+18.2 \log _{10}(f)+\alpha+\chi_{a}
$$

and the modified Highway condition is

$$
L_{s m}=23.4+20 \log _{10}(d)+20 \log _{10}(f)+\alpha+\chi_{a}
$$


TABLE I

DENSITY OF SAMPLES

\begin{tabular}{|c||c|}
\hline Sample No. & Density $\left(\mathrm{g} / \mathrm{m}^{3}\right)$ \\
\hline 1 & 2.5426 \\
2 & 2.56857 \\
3 & 2.6138 \\
4 & 2.62714 \\
5 & 2.4202 \\
6 & 2.9232 \\
7 & 2.4732 \\
8 & 2.5425 \\
9 & 2.4764 \\
\hline
\end{tabular}

where $\alpha$ is the attenuation factor of dust and sand that is defined by (4).

The proposed link margin $M_{d}$ is defined as

$$
M_{d}=\frac{G_{t} G_{r} P_{t}}{k T_{s} R L_{0} L_{s m}\left(\frac{E_{b}}{N_{0}}\right)}
$$

where $G_{r}$ is the receive antenna gain, $G_{t}$ is transmit antenna gain, $P_{t}$ is the transmitted power $(d B m), R$ is the data rate bps, $L_{0}$ is the circuit losses, $k$ is the Boltzmann's constant $1.38 \times 10^{-23} \mathrm{~J} / \mathrm{K}, T_{s}$ is the effective system noise temperature, $\frac{E_{b}}{N_{0}}$ is the required energy per bit to noise spectral density ratio, $L_{s m}$ is the modified free space path loss that is represented by (7) and (8).

\section{Field SAND ANd Dust Data}

The authors had field data from the desert of the southern part of Libya; thus, the region was identified as the study region to investigate the impact of dust and/or sand storms on the wireless communication systems [5], [11]. Moreover, this region is considered because it is characterized as a famous desert climate, and it has a fast wind filled with dust from time to time. Based on the study region information, nine places were chosen to collect the sand and dust carried by the wind. The plastic cans were placed on the tower or on the roof of buildings at the height of $13 \mathrm{~m}$ to collect dust and sand through the dust storms of different seasons (summer, fall, winter, and spring).

\section{A. Analysis of the samples}

For this study, samples' particle size distribution, average density, and chemical composition are required to compute the dielectric constant and attenuation factors. There are two laboratories available to carry out the analysis of the samples. These two laboratories are the Libyan Petroleum Institute and the Industrial research. The measured density of all samples which the Libyan Petroleum Institute has determined is shown in Table I.

The average density of all samples from Table I is equal to $2.5764 \mathrm{~g} / \mathrm{m}^{3}$. The size of dust and sand particles is determined using the sieving method. The sieving analysis showed that the major grain size of these samples ranges from less than $90 \mu \mathrm{m}$ to $600 \mu \mathrm{m}$. The complex permittivity of the composite component can be calculated using (2). The complex permittivity of each sample is shown in Table II.
TABLE II

Complex PermitTivity of EACH SAMPLE

\begin{tabular}{|c||c|}
\hline Sample No. & Complex Permittivity \\
\hline 1 & $5.0384-\mathrm{j} 0.0509$ \\
2 & $5.4851-\mathrm{j} 0.0562$ \\
3 & $5.4801-\mathrm{j} 0.0694$ \\
4 & $7.5929-\mathrm{j} 0.1140$ \\
5 & $6.7899-\mathrm{j} 0.1296$ \\
6 & $5.4003-\mathrm{j} 0.0787$ \\
7 & $7.4707-\mathrm{j} 0.1344$ \\
8 & $5.5713-\mathrm{j} 0.0704$ \\
9 & $8.3078-\mathrm{j} 0.1329$ \\
\hline
\end{tabular}

The average complex permittivity of the samples collected in the studied region from Table II is equal to $6.3485-j 0.0929$. The following empirical relation is used for the variation of complex permittivity with relative humidity [5]

$\epsilon_{1}=6.3485+0.04 H-7.78 \times 10^{-4} H^{2}+5.56 \times 10^{-6} H^{3}$

and

$\epsilon_{2}=0.0929+0.02 H-3.71 \times 10^{-4} H^{2}+2.76 \times 10^{-6} H^{3}$

where $H$ is the air relative humidity (percentage).

\section{RESUlTS AND DisCUSSION}

In this section, simulated numerical results and application from an unsignalized intersection are presented.

\section{A. Attenuation of Dust and Sand}

Equation (3) is used to estimate the attenuation of dust and sand in terms of concentration of dust, the particle size of sand, and frequency of the transmitted signal. The $5 \mathrm{G}(28 \mathrm{GHz})$ and DSCR $(5.9 \mathrm{GHz})$ are two bands of frequency. The particle size is changed from 0 to $538 \mu \mathrm{m}$. The maximum height is between $0.75 m-2 m$. The attenuation is computed at different values of humidity $(0 \%, 60 \%$, and $100 \%)$. Figure 1 shows the attenuation of dust and sand versus the visibility when the transmitted frequency is $5.9 \mathrm{GHz}$.

The dust and sand attenuation increases as the frequency increases, as shown in Fig. 2. Also, the same attenuation model is used to investigate the effect when the particle size is increased at different values of considered frequency. It is found that the value of the attenuation of $5 \mathrm{G}$ is higher than the value of DSCR as shown in Figs. 3 and 4.

\section{B. Link Budget of V2V}

The designed system consists of two vehicles. The sensor of every vehicle used a dual-band phased array antenna. This system is proposed and tested by a research team from Florida International University [22]. This dual-band RF front-end for DSRC and $5 \mathrm{G} \mathrm{V} 2 \mathrm{~V}$ operates at $5.9 \mathrm{GHz}$ and $28 \mathrm{GHz}$. The modified link budget of the $\mathrm{V} 2 \mathrm{~V}$ communication link that is presented in (9) is used to estimate the received power signal. If the received energy per bit to noise spectral density ratio is achieving the required bit error rate, the communication between $\mathrm{V} 2 \mathrm{~V}$ is reliable. On the contrary, if the received 


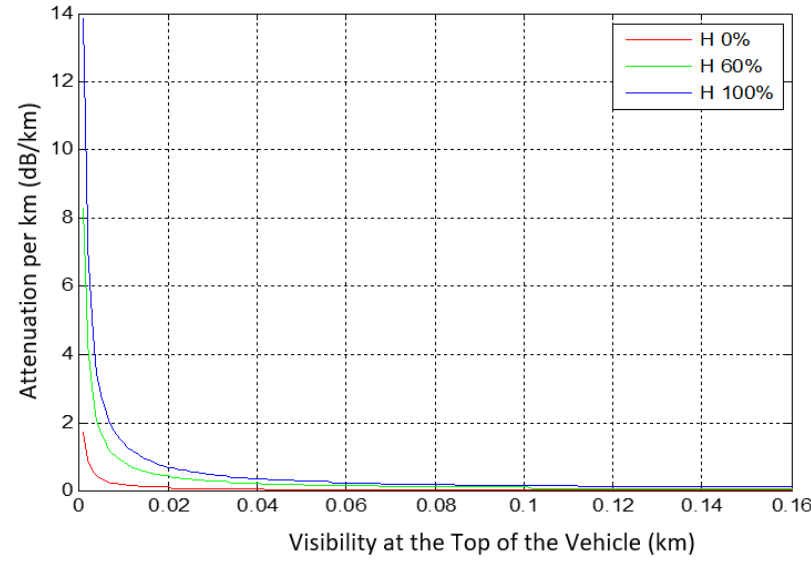

Fig. 1. Attenuation versus the visibility at $5.9 \mathrm{GHz}$ and $h=1 \mathrm{~m}$

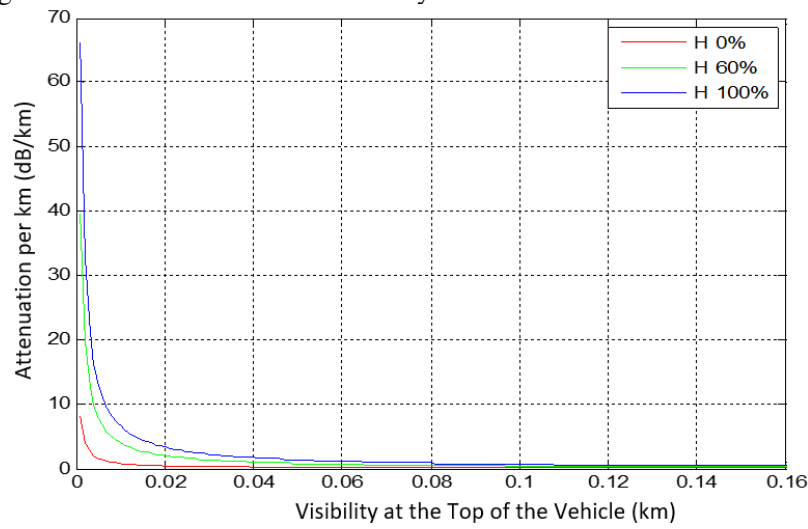

Fig. 2. Attenuation versus the visibility at $28 \mathrm{GHz}$ and $h=1 \mathrm{~m}$

TABLE III

REQUIRED LINK BUDGET INFORMATION

\begin{tabular}{|c|c|c|}
\hline & DSRC 5.9GHz & mm-wave 28GH \\
\hline Transmit Antenna Gain & $9.9 \mathrm{dBi}$ & $23.4 \mathrm{dBi}$ \\
Receive Antenna Gain & $9.9 \mathrm{dBi}$ & $23.4 \mathrm{dBi}$ \\
Transmit power & $27 \mathrm{dBm}$ & $27 \mathrm{dBm}$ \\
EIRP & $36.9 \mathrm{dBm}$ & $50.4 \mathrm{dBm}$ \\
Required & $18.8 \mathrm{~dB}$ & $18.8 \mathrm{~dB}$ \\
Data Rate & $27 \mathrm{Mbps}$ & $1 \mathrm{Gbps}$ \\
\hline
\end{tabular}

energy per bit to noise spectral density ratio is not achieving the required bit error rate, the communication between $\mathrm{V} 2 \mathrm{~V}$ is disconnected.

Link Budget information of $5.9 \mathrm{GHz}$ and $28 \mathrm{GHz}$ is shown in Table III [22].

According to the proposed model of Sandhiya Reddy Govindarajulu and Elias A. Alwan [22], it assumed the overall circuit losses $L_{0}=5 d B$, receiver front-end noise figure $F=6 d B, T_{s}=T_{A}+(F-1) 290, T_{A}=290 K$, the distance between vehicles $d=390 \mathrm{~m}$ and $\frac{E_{b}}{N_{0}}=18.8 d B$ that is required to achieve a bit error rate $B E R$ equal to $10^{-6}$. Moreover, the radio frequency parameters that are presented in Table III are used together to insert into (9) to calculate the value of $L_{s m}$ to maintain the link margin $M_{d} \geq 10 d B$. If the $M_{d} \geq 10 d B$, all the required transmission parameters are

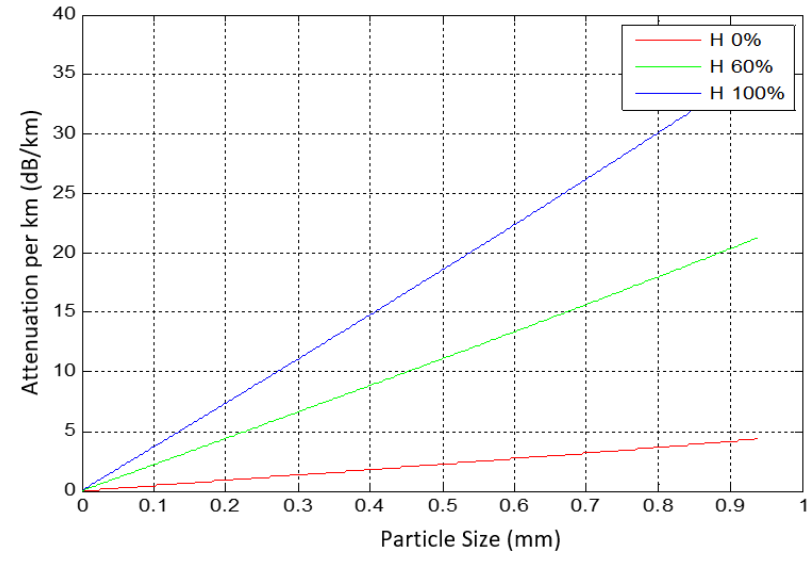

Fig. 3. Attenuation versus the particle size $(\mathrm{mm})$ at $5.9 \mathrm{GHz}$ and $h=1 \mathrm{~m}$

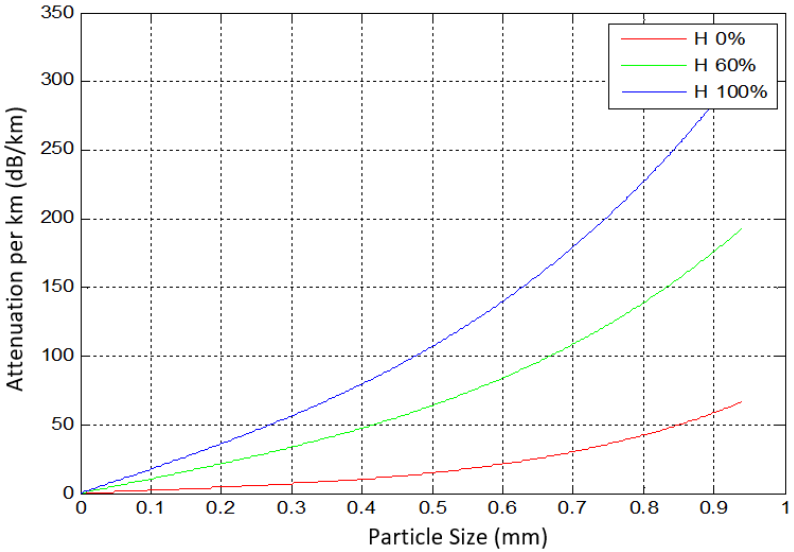

Fig. 4. Attenuation versus the particle size $(\mathrm{mm})$ at $28 \mathrm{GHz}$ and $h=1 \mathrm{~m}$

achieved to avoid any disconnect or loss of communication between autonomous vehicles. Since the attenuation $L_{s m}$ at $M_{d}=10 d B$ (the threshold value) is computed, Figs. 1-4 are used to identify the minimum equivalent particle radius and visibility (concentration of dust) to avoid communication loss between V2V. In other words, these minimum values of equivalent particle radius and visibility are the threshold value. If the equivalent particle radius increases or the visibility decreases, the communication between V2V will discount. For this reason, this research is important to design a reliable system to support the recent intelligent transportation network. In this simulation, $5 \mathrm{G}$ communication channel $(28 \mathrm{GHz})$ and DSRC channel $(5.9 \mathrm{GHz})$ are considered to investigate the effect of dust and sand. The result of this simulation is summarized in Table IV.

Table IV shows that dust and sand affect the 5G communication channel more than the DSRC channel. This effect is logical because the wavelength $\lambda$ of the propagating $5 \mathrm{G} \mathrm{mm}$ wave is short compared to the particle size of dust and sand. It is seen that the $5.9 \mathrm{GHz}$ is not affected by the dust in the dry weather $(H=0 \%)$ for both scenarios, but the $5 \mathrm{G}(28 \mathrm{GHz})$ is affected when visibility is less than $51 \mathrm{~m}$ in the highway case. At the $\mathrm{H}=0 \%$, the $5.9 \mathrm{GHz}$ is affected if the particle size is greater than $1.48 \mathrm{~mm}$ in the urban case and $614 \mu \mathrm{m}$ in the highway case. At the $H=0 \%$, the $28 \mathrm{GHz}$ is affected if the 
TABLE IV

SIMULATION RESULTS

\begin{tabular}{|c|c|c|c|}
\hline Humidity & Frequency & Urban & Highway \\
\hline $0 \%$ & $\begin{array}{l}5.9 \mathrm{GHz} \\
28 \mathrm{GHz}\end{array}$ & $\begin{array}{l}\mathrm{V} \text { is not affected } \\
P S \geq 1.48 \mathrm{~mm} \\
\mathrm{~V} \text { is not affected } \\
P S \geq 0.27 \mathrm{~mm}\end{array}$ & $\begin{array}{c}\mathrm{V} \text { is not affected } \\
P S \geq 614 \mu \mathrm{m} \\
\mathrm{V} \leq 51 \mathrm{~m} \\
P S \geq 2 \mu \mathrm{m}\end{array}$ \\
\hline $60 \%$ & $\begin{array}{l}5.9 \mathrm{GHz} \\
28 \mathrm{GHz}\end{array}$ & $\begin{array}{c}\mathrm{V} \text { is not affected } \\
P S \geq 0.328 \mathrm{~mm} \\
\mathrm{~V} \leq 3 \mathrm{~m} \\
P S \geq 58 \mu \mathrm{m}\end{array}$ & $\begin{array}{c}\mathrm{V} \leq 2 \mathrm{~m} \\
P S \geq 129 \mu \mathrm{m} \\
\mathrm{V} \leq 120 \mathrm{~m} \\
P S \geq 0.39 \mu \mathrm{m}\end{array}$ \\
\hline $100 \%$ & $\begin{array}{l}5.9 \mathrm{GHz} \\
28 \mathrm{GHz}\end{array}$ & $\begin{array}{c}\mathrm{V} \leq 1 \mathrm{~m} \\
P S \geq 0.2 \mathrm{~mm} \\
\mathrm{~V} \leq 5 \mathrm{~m} \\
P S \geq 36 \mu \mathrm{m}\end{array}$ & $\begin{array}{c}V \leq 3 \mathrm{~m} \\
P S \geq 70 \mu \mathrm{m} \\
V \leq 160 \mathrm{~m} \\
P S \geq 0.2 \mu \mathrm{m}\end{array}$ \\
\hline
\end{tabular}

particle size is greater than $0.27 \mathrm{~mm}$ in the urban case and 2 $\mu m$ in the highway case.

At the $H=60 \%$, the $5.9 \mathrm{GHz}$ is affected if the particle size is greater than $0.328 \mathrm{~mm}$ in the urban case and $129 \mu \mathrm{m}$ in the highway case, but it is affected by the concentration of dust if the visibility is less than $2 \mathrm{~m}$ in the highway case. At the $H=60 \%$, the $28 \mathrm{GHz}$ is affected if the particle size is greater than $58 \mu \mathrm{m}$ and the visibility less than $0.39 \mu \mathrm{m}$ in the urban case. It is affected when the particle size is greater than and the visibility less than $120 \mathrm{~m}$ in the highway case.

At the $H=100 \%$, the $5.9 \mathrm{GHz}$ is affected if the particle size is greater than $0.2 \mathrm{~mm}$ in urban case and $70 \mu \mathrm{m}$ in the highway case. Also, it is affected by the concentration of dust if the visibility is less than $1 \mathrm{~m}$ in the urban scenario and 3 $m$ in the highway scenario. At the $H=100 \%$, the $28 \mathrm{GHz}$ is affected if the particle size is greater than $36 \mu \mathrm{m}$ and the visibility less than $5 \mathrm{~m}$ in the urban case. Also, it is affected when the particle size is greater than $0.2 \mu \mathrm{m}$ and the visibility less than $160 \mathrm{~m}$ in the highway case.

\section{Factors Affecting Packet Loss in V2V}

In order to analyze the impact of dust and sand on received packets among connected vehicles, a simple 2-vehicle communication scenario is simulated. The simulation is developed on NS-3, which is a discrete-event network simulator for Internet systems. An additional NS-3 module Millicar [15] is used to simulate the mm-Wave-based V2X networks. This module enables end-to-end, full-stack simulations of vehicular networks with a 3GPP channel model for $\mathrm{V} 2 \mathrm{~V}$ propagation and fading at mm-Waves, and physical and MAC layers redesigned for NR V2X. The Millicar module is redefined and the weather impact factor $A_{d}$ is added to quantify the pathloss. A simple vehicle-to-vehicle (V2V) scenario is generated with the proposed pathloss involved 5G NR channel model. In our proposed simulation scenario, two connected vehicles are placed apart at a distance of $10 \mathrm{~m}$ and moving at the same speed of $20 \mathrm{~m} / \mathrm{s}$ to the same direction. The vehicles exchange packets through a UDP application using a wireless channel. Packet size is set to 1024 and bandwidth is $0.1 \mathrm{GHz}$.

The pathloss function considers the weather impact by involving several parameters, and these parameters are subject to change to demonstrate the weather impact on the communication channels.
TABLE V

Simulation PARAMETERS

\begin{tabular}{|c|c|}
\hline Parameters & Value range \\
\hline Particle Size $(\mathrm{m})$ & $0.0001-0.0004$ \\
Visibility (km) & $0.0006-0.003$ \\
Humidity (\%) & $0 \%-100 \%$ \\
Frequency $(\mathrm{GHz})$ & $28,73.5$ \\
Speed (m/s) & $1-40$ \\
Inter packet interval (microseconds) & $1-35$ \\
V2V scenarios & Highway, Urban \\
Vehicle states & Line-of-Sight, Non-Line-of-Sight, \\
& Vehicle Non-Line-of-Sight \\
\hline
\end{tabular}

In the first series of simulations, all the parameter impacts in weather conditions $A_{d}$ are tested in the Millicar module. As shown in Table V, the factors of particle size, visibility, humidity, frequency, vehicle speed, and inter packet Interval are examined changing one factor at a time to understand how they affect communications between two vehicles.

\section{Particle Size, Visibility, Humidity and Frequency}

According to Fig. 5, when particle size is greater than $0.2 \mathrm{~mm}$, the received packets between two vehicles decrease drastically as the particle size increases. When particle size is greater than $0.35 \mathrm{~mm}$, all the packets between two vehicles would be dropped to zero, which means that there would be no communication between the two vehicles.

Figure 6 shows that as visibility increases, the packets between two vehicles also increase. In more detail, when visibility is less than $1 \mathrm{~m}$, there is no communication between the two vehicles. Starting from visibility of $1 \mathrm{~m}$, the received packets increase significantly as the visibility increases and all the packets between two vehicles would be received when visibility increases to $2 \mathrm{~m}$.

Figure 7 shows the effect of humidity. When humidity is less than $60 \%$, the received packets keeps the same level and it starts to fall rapidly when humidity passes the $60 \%$ threshold.

Figure 8 indicates that the packets between two vehicles will decrease as we increase frequency and $45 \mathrm{GHz}$ could be the threshold for no communication under these settings.

\section{E. Vehicle speed and inter packet interval}

Figures 9 and 10 reveal the impact of vehicle speed and inter packet interval, which are two important parameters in Millicar module. Based on the results, these two parameters do not have considerable influence on the mm-Wave communications between two vehicles, and thus are ignored in next sections.

\section{F. Compare packets loss with free space path loss}

To check the relationship between simulation results and calculated free space path loss introduced in Section III, received packets and path loss are shown in the same graph and to identify the trend change.

According to the results, there exists a threshold for the path loss such that no packets would be received if the path loss is larger than this threshold (in this case, visibility reaches a small enough value in Fig. 11, or particle size reaches a 


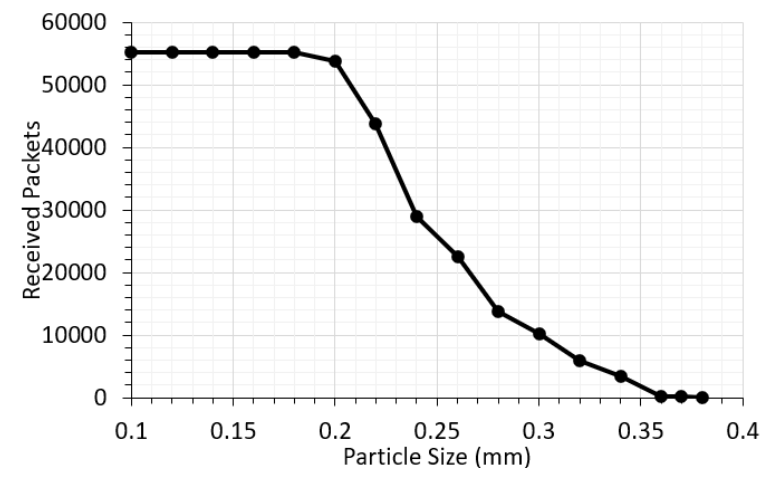

Fig. 5. Impact of particle size $(\mathrm{mm})$ on received packets

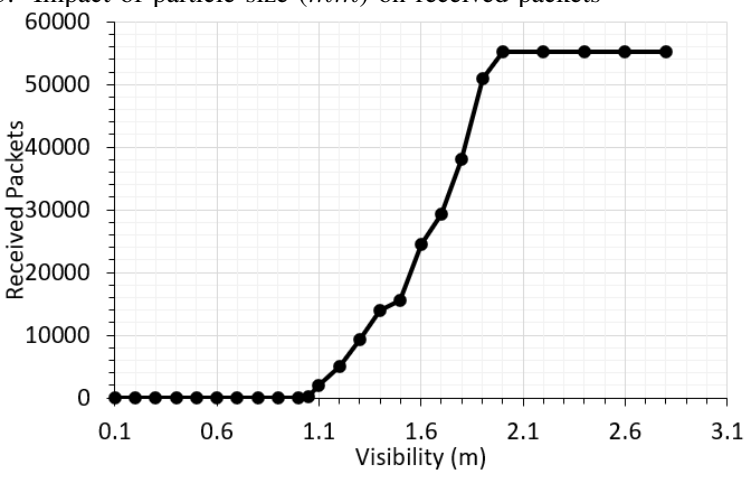

Fig. 6. Impact of visibility $(m)$ on received packets

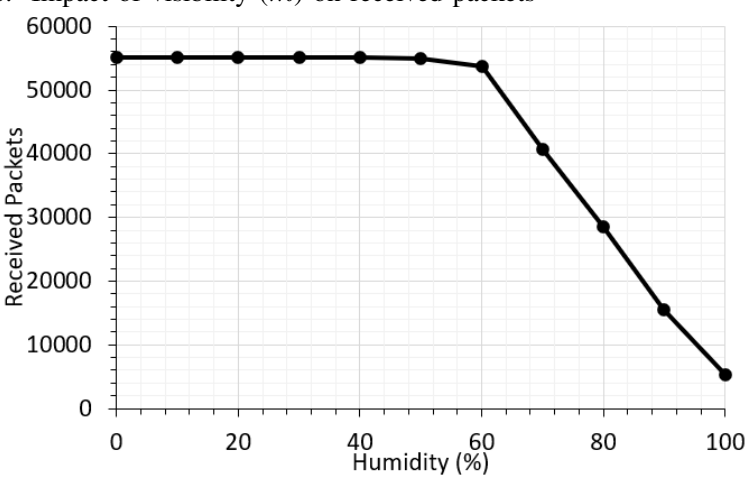

Fig. 7. Impact of humidity (\%) on received packets

large enough value in Fig. 12). And, this threshold value changes under different operating frequency and humidity conditions. In Fig. 11, as visibility increases, the received packets increases whereas the path loss decreases.

On the contrary, the particle size shows the opposite effect in Fig. 12. Specifically, as the particle size increases, the received packets decreases whereas the path loss increases.

As dust and sand have shown to be able to affect the received packets in section $\mathrm{V}(\mathrm{C})$, a more realistic scenario is created in NS-3 to analyze their actual impact on the behavior of the connected vehicles. In this intersection collision warning scenario, 2-vehicle are placed at their own start point in the four-way intersection (Fig. 13). The initial distance between each vehicle and the crossing is $40 \mathrm{~m}$ and the initial speed is set to $20 \mathrm{~m} / \mathrm{s}$ for each. Without the intersection collision warning system, the 2-vehicle would arrive at the crossing

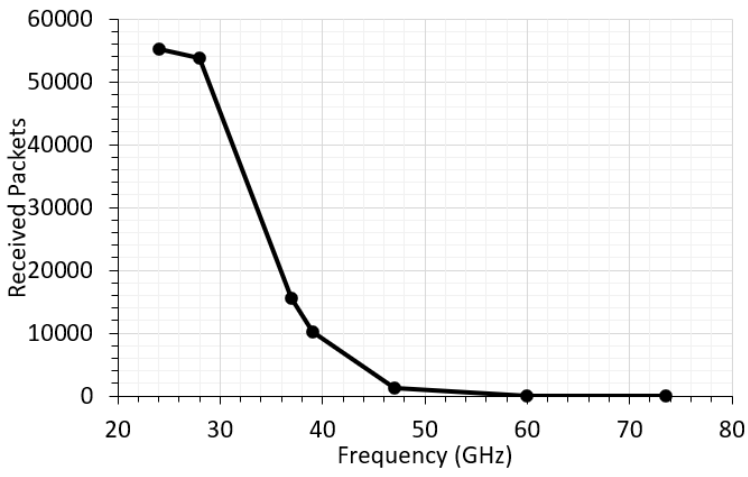

Fig. 8. Impact of frequency on received packets

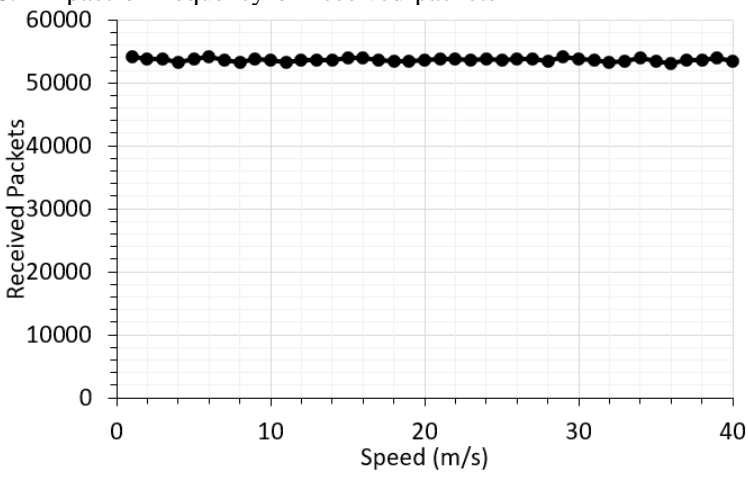

Fig. 9. Impact of vehicle speed $(\mathrm{m} / \mathrm{s})$ on received packets

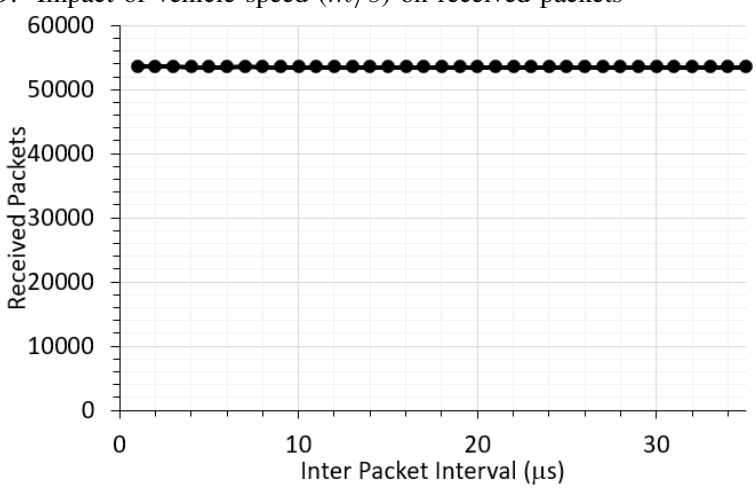

Fig. 10. Impact of inter packet times $(\mu s)$ on received packets

simultaneously and there is a possibility of collision. After enabling the intersection collision warning system, one vehicle would receive the estimated time-to-collision information from the other vehicle and would thus reduce its speed to avoid the collision. The vehicles exchange packets through a UDP application using a wireless channel. Packet size is set to 1024 and bandwidth is $0.1 \mathrm{GHz}$. The details of the selection of parameters are shown in Table VI.

As shown in Fig. 14, compared with $0 \%$ and $60 \%$ humidity, when humidity is $100 \%$, the collision distance starts to decline rapidly at a smaller particle size of $0.18 \mathrm{~mm}$. The corresponding particle size is $0.28 \mathrm{~mm}$ for $60 \%$ humidity and $7 \mathrm{~mm}$ for $0 \%$ humidity.

As shown in Fig. 15, under higher frequency of $73.5 \mathrm{GHz}$, when the humidity is $100 \%$, the two vehicles would always collide at the intersection no matter how particle size changes. 

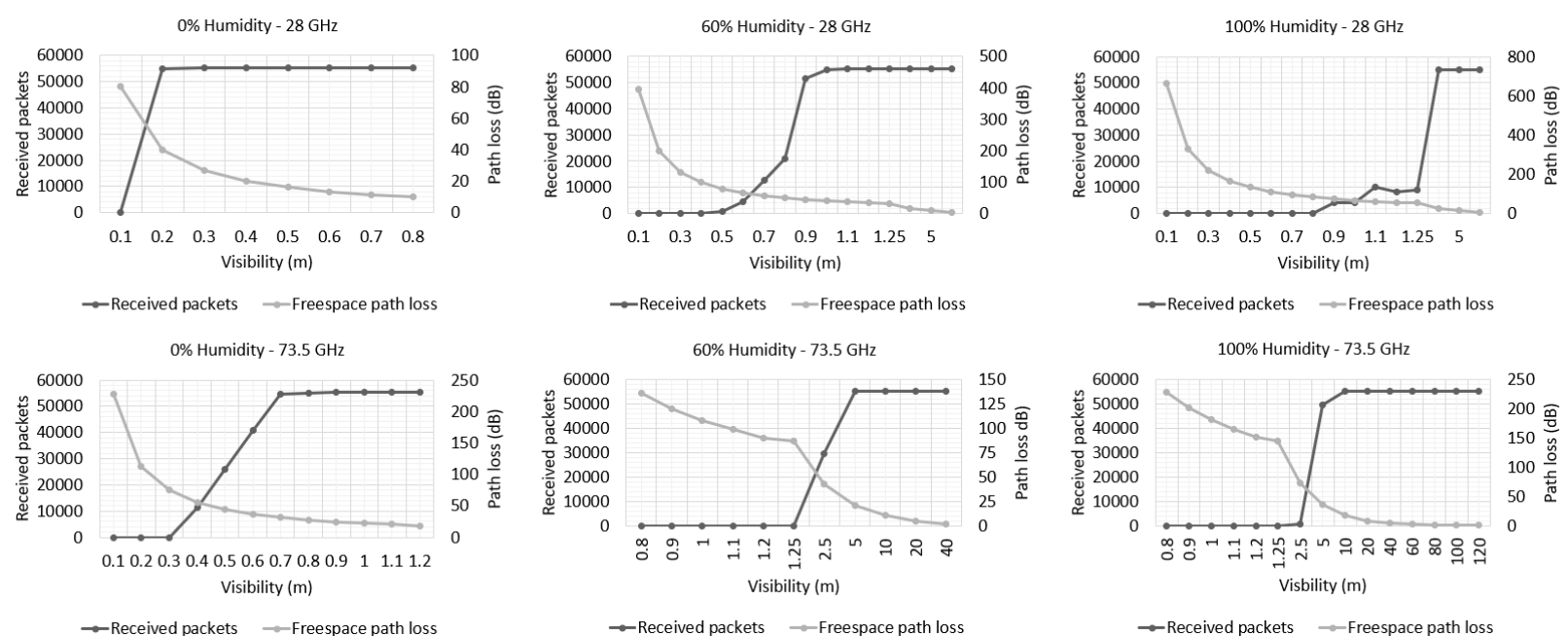

Fig. 11. Impact of visibility on the received packets and path loss under different humidity conditions and operating frequency
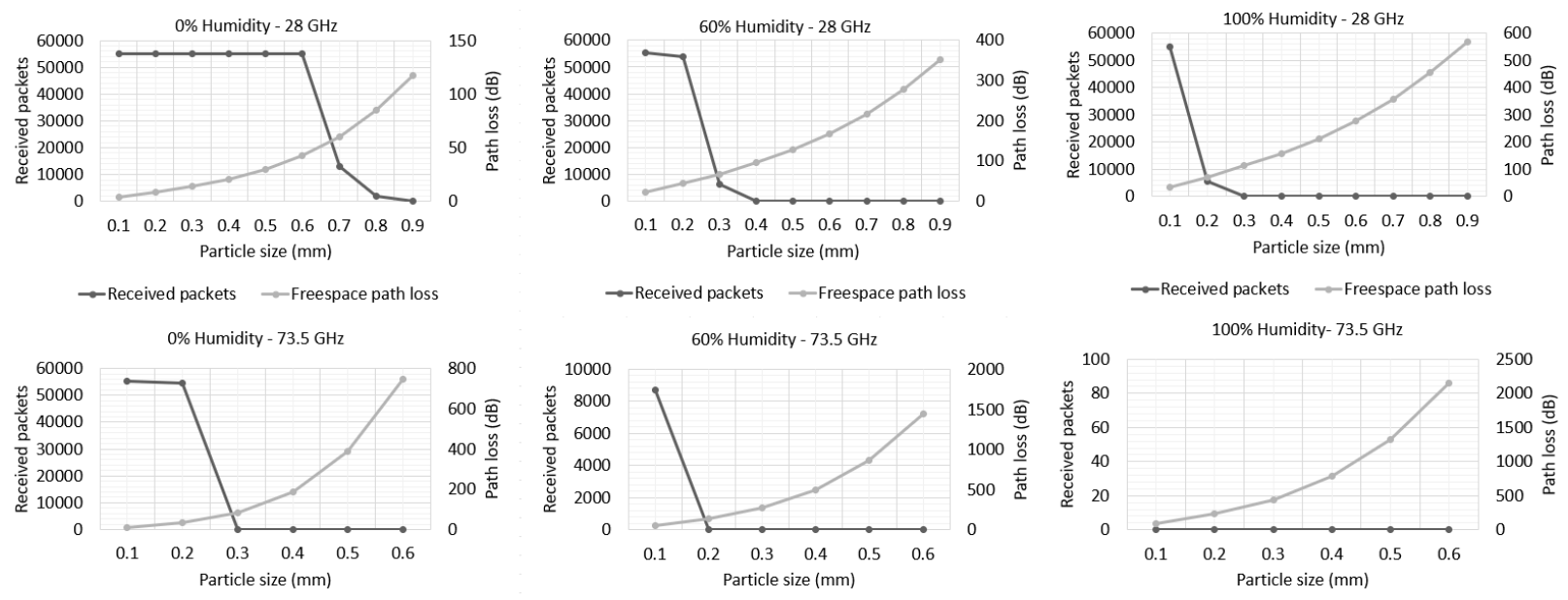

$\rightarrow$ Received packets $\rightarrow$ Freespace path loss

$\rightarrow$ Received packets $\rightarrow$ Freespace path loss

$\rightarrow$ Received packets $\rightarrow$ Freespace path loss

Fig. 12. Impact of particle size on the received packets and path loss under different humidity conditions and operating frequency.

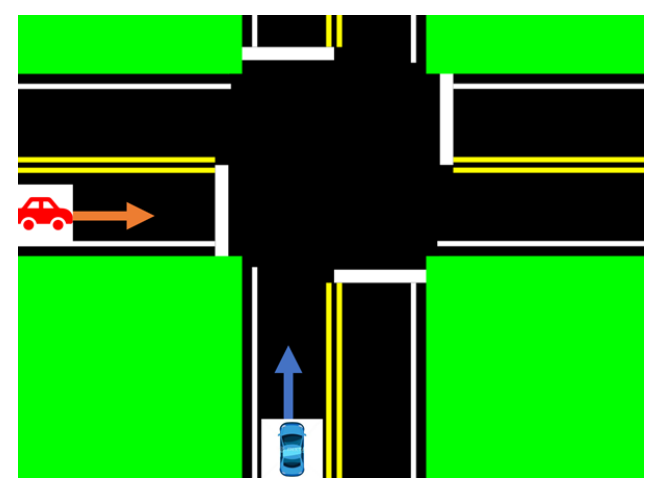

Fig. 13. Snapshot of the intersection simulated

Similarly, under $60 \%$ humidity level, when the particle size is greater than $0.14 \mathrm{~mm}$, the two vehicles will crash as well. Under $0 \%$ humidity, the two vehicles would be safe until particle size is increased to $0.3 \mathrm{~mm}$.

Comparing results in Figs. 16 and 17, under all three
TABLE VI

SimUlation PARAMETERS

\begin{tabular}{|c|c|}
\hline Parameters & Value range \\
\hline Particle Size $(\mathrm{m})$ & $0.0001-0.0009$ \\
Visibility $(\mathrm{km})$ & 0.002 \\
Humidity $(\%)$ & $0 \%, 60 \%, 100 \%$ \\
Frequency $(\mathrm{GHz})$ & $28,73.5$ \\
Speed $(\mathrm{m} / \mathrm{s})$ & 20 \\
& $(15$ after receiving signals $)$ \\
Inter packet interval $(\mu s)$ & 30 \\
V2V scenarios & Urban \\
Vehicle states & Non-Line-of-Sight \\
\hline
\end{tabular}

humidity conditions, the corresponding visibility of collision distance starts to increase are $0.2 \mathrm{~m}, 1 \mathrm{~m}, 2 \mathrm{~m}$ for $28 \mathrm{GHz}$ and $0.9 \mathrm{~m}, 3.5 \mathrm{~m}, 6 \mathrm{~m}$ for $73.5 \mathrm{GHz}$. Higher frequency increases the weather impact which results in better visibility to enable our collision warning system.

\section{CONCLUSION}

In this research, the effect of dust and sand storms on the $5 \mathrm{G}$ wireless communication channel between connected vehicles 


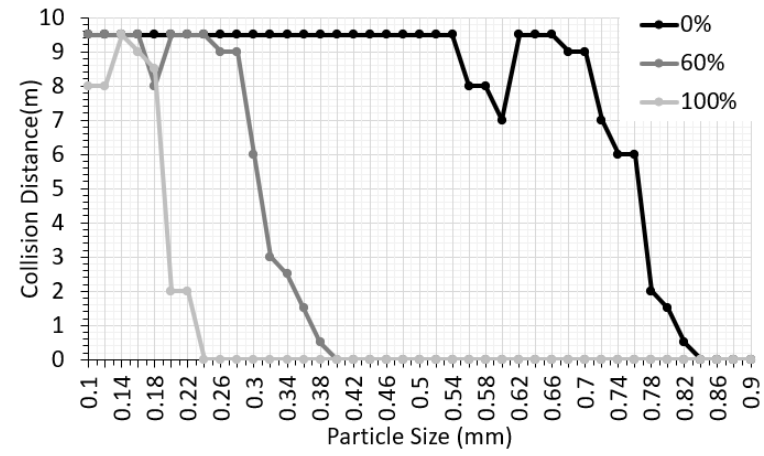

Fig. 14. Relationship between particle size and distance under different humidity for $28 \mathrm{GHz}$

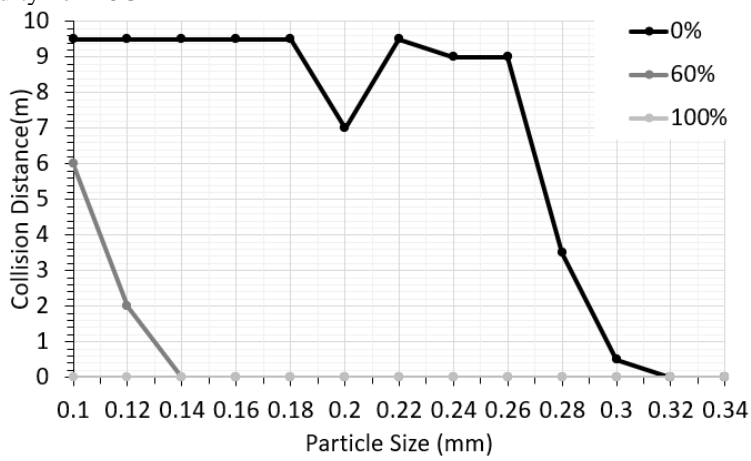

Fig. 15. Relationship between particle size and distance under different humidity for $73.5 \mathrm{GHz}$

was investigated. The proposed margin link model is used to simulate the effect of dust and sand. The main object point of this work is to find the threshold value of particle size of sand and the concentration to avoid the loss of communication between autonomous vehicles. The simulation results show that the attenuation of the $5 \mathrm{G}$ propagating signal increases when the operating frequency, the concentration of the dust, and the particle size of sand are increased. Also, it is seen that the $5 \mathrm{G} \mathrm{mm}$-wave communication channel is more affected by dust and sand storms than the DSRC channel.

For future work, this proposed model would be validated by using real measurements. Moreover, the real measurement of the dielectric constant, particle size range and concentration of dust of the desert region in the United States would be considered to figure out the values of real attenuation of dust and sand for this specific region.

\section{ACKNOWLEDGMENTS}

This study is partially supported by the Center for Connected Multimodal Mobility $\left(C^{2} M^{2}\right.$ ) (USDOT Tier 1 University Transportation Center) headquartered at Clemson University, Clemson, SC. Any opinions, findings, and conclusions or recommendations expressed in this paper are those of the authors and do not necessarily reflect the views of $C^{2} M^{2}$ and the official policy or position of the USDOT/OST-R, or any State or other entity, and the U.S. Government assumes no liability for the contents or use thereof. It is also partially supported

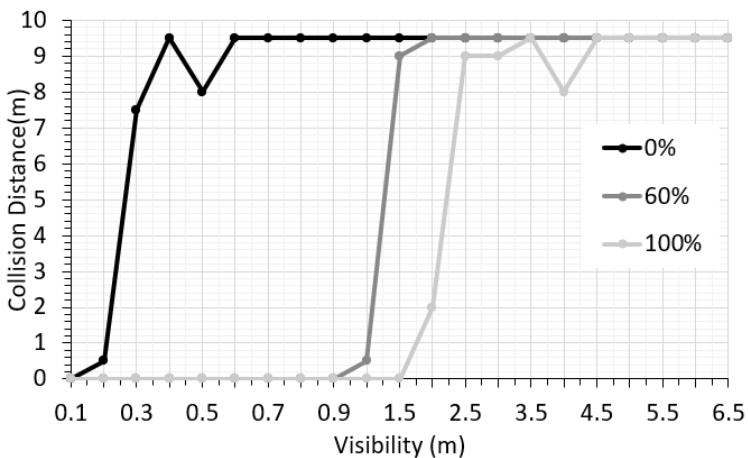

Fig. 16. Relationship between visibility and collision distance under different humidity for $28 \mathrm{GHz}$

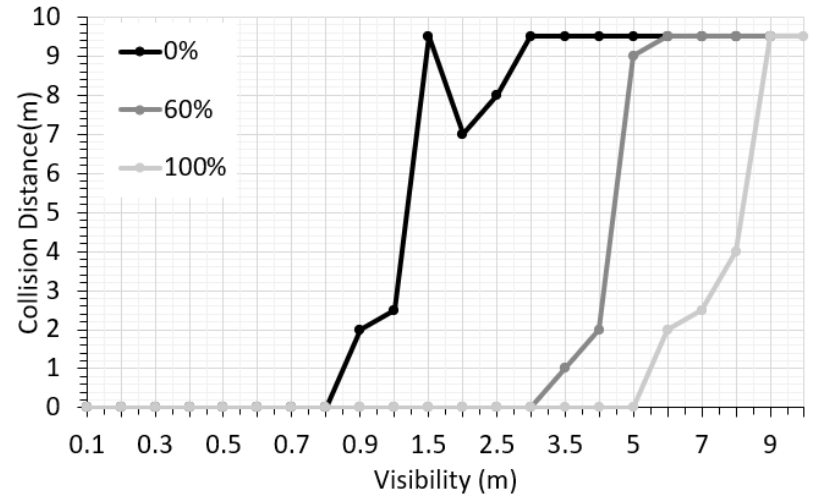

Fig. 17. Relationship between visibility and collision distance under different humidity for $73.5 \mathrm{GHz}$

by U.S. Department of Energy-National Nuclear Security Administration (NNSA) PuMP, MSIPP IAM-EMPOWEREd, MSIPP, Department of Education MSEIP programs, NASA ULI (University of South Carolina-Lead), and NSF Grant Nos. 1719501, 1954532, and 2131080.

\section{REFERENCES}

[1] E. M. Abuhdima, G. Comert, P. Pisu, C.-T. Huang, A. Elqaouaq, C. Zhao, S. Alston, K. Ambrose, and J. Liu, "The effect of dust and sand on the $5 \mathrm{~g}$ millimeter-wave links," in 2021 IEEE International Conference on Wireless for Space and Extreme Environments (WiSEE). IEEE, 2021, pp. 60-65.

[2] S. Zang, M. Ding, D. Smith, P. Tyler, T. Rakotoarivelo, and M. A. Kaafar, "The impact of adverse weather conditions on autonomous vehicles: how rain, snow, fog, and hail affect the performance of a selfdriving car," IEEE vehicular technology magazine, vol. 14, no. 2, pp. 103-111, 2019.

[3] R. He, A. F. Molisch, F. Tufvesson, Z. Zhong, B. Ai, and T. Zhang, "Vehicle-to-vehicle propagation models with large vehicle obstructions," IEEE Transactions on Intelligent Transportation Systems, vol. 15, no. 5, pp. 2237-2248, 2014.

[4] S. M. Khan, M. Chowdhury, M. Rahman, and M. Islam, "Feasibility of $5 \mathrm{~g}$ mm-wave communication for connected autonomous vehicles," arXiv preprint arXiv:1808.04517, 2018.

[5] E. M. Abuhdima and I. M. Saleh, "Effect of sand and dust storms on microwave propagation signals in southern libya," in Melecon 2010-2010 15th IEEE Mediterranean Electrotechnical Conference. IEEE, 2010, pp. 695-698.

[6] M. Giordani, T. Shimizu, A. Zanella, T. Higuchi, O. Altintas, and M. Zorzi, "Path loss models for $\mathrm{v} 2 \mathrm{v}$ mmwave communication: performance evaluation and open challenges," in 2019 IEEE 2nd Connected and Automated Vehicles Symposium (CAVS). IEEE, 2019, pp. 1-5. 
[7] M. Yang, B. Ai, R. He, L. Chen, X. Li, Z. Huang, J. Li, and C. Huang, "Path loss analysis and modeling for vehicle-to-vehicle communications with vehicle obstructions," in 2018 10th International Conference on Wireless Communications and Signal Processing (WCSP). IEEE, 2018, pp. 1-6.

[8] B. Kihei, J. A. Copeland, and Y. Chang, "Improved $5.9 \mathrm{ghz} v 2 \mathrm{v}$ short range path loss model," in 2015 IEEE 12th International Conference on Mobile Ad Hoc and Sensor Systems. IEEE, 2015, pp. 244-252.

[9] A. M. Al-Saman, M. Cheffena, M. Mohamed, M. H. Azmi, and Y. Ai, "Statistical analysis of rain at millimeter waves in tropical area," IEEE Access, vol. 8, pp. 51 044-51 061, 2020.

[10] J. Lee and B. Park, "Development and evaluation of a cooperative vehicle intersection control algorithm under the connected vehicles environment," IEEE Transactions on Intelligent Transportation Systems, vol. 13, no. 1, pp. 81-90, 2012.

[11] E. M. Abuhdima and I. M. Saleh, "Effect of sand and dust storms on gsm coverage signal in southern libya," in 2010 International Conference on Electronic Devices, Systems and Applications. IEEE, 2010, pp. 264 268.

[12] S. Sun, T. S. Rappaport, M. Shafi, P. Tang, J. Zhang, and P. J. Smith, "Propagation models and performance evaluation for $5 \mathrm{~g}$ millimeter-wave bands," IEEE Transactions on Vehicular Technology, vol. 67, no. 9, pp. 8422-8439, 2018

[13] MathWorks, "Matlab 5g toolbox," 2021. [Online]. Available: https: //www.mathworks.com/products $/ 5 \mathrm{~g} . \mathrm{html}$

[14] G. Nardini, D. Sabella, G. Stea, P. Thakkar, and A. Virdis, "Simu5g-an omnet++ library for end-to-end performance evaluation of $5 \mathrm{~g}$ networks," IEEE Access, vol. 8, pp. 181 176-181 191, 2020.

[15] M. Drago, T. Zugno, M. Polese, M. Giordani, and M. Zorzi, "Millicar: An ns-3 module for mmwave nr v2x networks," in Proceedings of the 2020 Workshop on $n s-3,2020$, pp. 9-16.

[16] Y. Youlin, V. Squires, and L. Qi, "Global alarm: dust and sandstorms from the world's drylands," in United Nations Convention to Combat Desertification (UNCCD). United Nations, 2002.

[17] I. M. Saleh, H. M. Abufares, and H. M. Snousi, "Estimation of wave attenuation due to dust and sand storms in southern libya using mie model," in WAMICON 2012 IEEE Wireless \& Microwave Technology Conference. IEEE, 2012, pp. 1-5.

[18] S. Ghobrial and S. Sharief, "Microwave attenuation and cross polarization in dust storms," IEEE transactions on antennas and propagation, vol. 35, no. 4, pp. 418-425, 1987.

[19] S. Sharief and S. Ghobrial, "X-band measurements of the dielectric constant of dust," in Proc. Ursi Commission F, 1983, pp. 143-147.

[20] S. M. Sharif, "Attenuation properties of dusty media using mie scattering solution," Progress In Electromagnetics Research M, vol. 43, pp. 9-18, 2015.

[21] A. Musa and B. S. Paul, "Prediction of electromagnetic wave attenuation in dust storms using mie scattering," in 2017 IEEE AFRICON. IEEE, 2017, pp. 603-608.

[22] S. R. Govindarajulu and E. A. Alwan, "Range optimization for dsrc and 5g millimeter-wave vehicle-to-vehicle communication link," in 2019 International Workshop on Antenna Technology (iWAT). IEEE, 2019, pp. $228-230$.

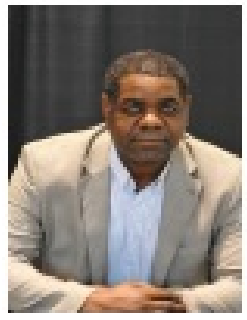

Esmail Abuhdima received the B.Sc. and M.Sc degree in Electrical and Electronic Engineering from Tripoli University, Tripoli, Libya and the Ph.D. degree in Electrical Engineering from University of Dayton, Dayton, Ohio, in 1998, 2009, and 2017 respectively. He is currently with Computer Science, Physics and Engineering Department, Benedict College, Columbia, SC. His research interests include Wave Propagation, Simulation of Radar Signals, Antenna and Electromagnetic Field Theory, RF design and systems.He serves as an Editorial Board Members in American Journal of Electromagnetics and Applications(AJEA). Also, he is a reviewer in Advances in Science, Technology and Engineering Systems Journal (ASTESJ).

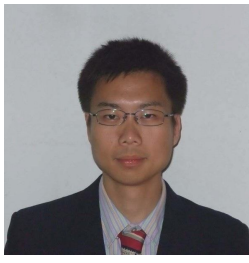

Jian Liu received his B.S. degree in Applied Chemistry from Tianjin University, China. He received one M.S. degree in Statistics and one M.S. degree in Computer Science from West Virginia University, USA in 2018. Jian is now a Ph.D. student in the Department of Computer Science and Engineering, University of South Carolina. His research focuses on network transmission and security. $\mathrm{He}$ is also interested in cloud and edge computing and blockchain.

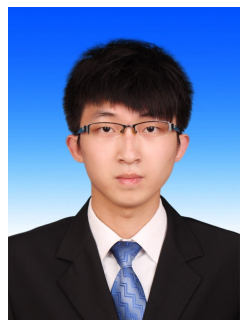

Chunheng Zhao received the B.E. degree in Automotive Engineering from Harbin Institute of Technology, China, in 2016, and the M.S. degree in Automotive Engineering from Clemson University, in 2019. He is currently pursuing a Ph.D. degree in Automotive Engineering at Clemson University International Center for Automotive Research. His research interests include functional safety and security issues in connected and automated vehicles. $\mathrm{He}$ is also interested in deep learning-based perception and control in autonomous driving.

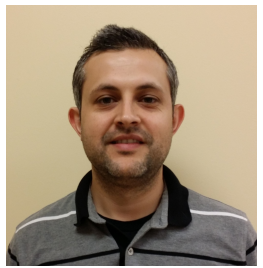

Gurcan Comert received the B.Sc. and M.Sc. degree in Industrial Engineering from Fatih University, Istanbul, Turkey and the Ph.D. degree in Civil Engineering from University of South Carolina, Columbia, SC, in 2003, 2005, and 2008 respectively. He is currently with Physics and Engineering Department, Benedict College, Columbia, SC. His research interests include applications of statistical models to transportation problems, traffic parameter prediction, and stochastic models.

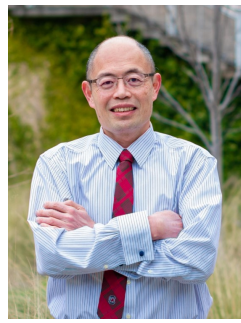

Chin-Tser Huang received the B.S. degree in Computer Science and Information Engineering from National Taiwan University, Taipei, Taiwan, in 1993 , and the M.S. and Ph.D. degrees in Computer Sciences from The University of Texas at Austin in 1998 and 2003, respectively. He is a Professor in the Department of Computer Science and Engineering at University of South Carolina at Columbia. His research interests include network security, network protocol design and verification, and distributed systems. He is the director of the Secure Protocol Implementation and Development (SPID) Laboratory at the University of South Carolina. He is the author (along with Mohamed Gouda) of the book "Hop Integrity in the Internet," published by Springer in 2005. His research has been funded by DARPA, AFOSR, AFRL, NSF, NEH, and USDOT. He is an NRC Research Associate in 2020, and a recipient of the USAF Summer Faculty Fellowship Award and of the AFRL Visiting Faculty Research Program Award in 2008-2021. He is a Senior Member of IEEE and ACM. 


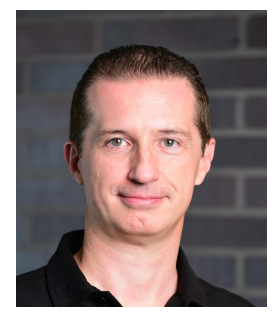

Pierluigi Pisu is a Professor of Automotive Engineering in the Carroll A. Campbell Jr. Graduate Engineering Center at the Clemson University International Center for Automotive Research with a joint appointment in the Holcombe Department of Electrical and Computer Engineering at Clemson University. He has a Ph.D. in Electrical Engineering from The Ohio State University (2002) and a M.S. in Computer Engineering from the University of Genoa, Italy. His research interests lie in functional safety, security, control and optimization of CyberPhysical Systems for next generation of high performance and resilient connected and automated systems with emphasis in both theoretical formulation and virtual/hardware-in-the-loop validation. He published 50 journal and 100 conference papers; he holds 3 patents and published a book on "Fault Detection and Isolation with Applications to Vehicle Systems".

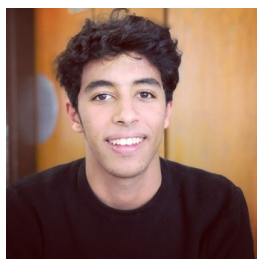

Ahmed Elqaouaq is a senior undergraduate Student in Electrical Engineering at Benedict College, Columbia, SC. His research team is working on the Impact of Weather factor on $5 \mathrm{G}$ communication Channel under Connected Vehicles Framework.

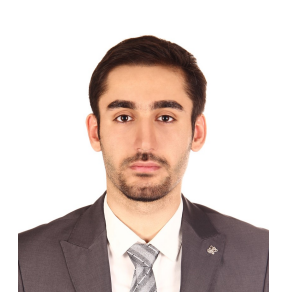

Amirhossein Nazeri received his BSc and MSc degrees in the Electrical and Computer Engineering from the Iran University of Science and Technology, and Texas Tech University, respectively. He is currently working towards his $\mathrm{PhD}$ in Automotive Engineering department at Clemson University. His research interests lies in Machine Learning and Deep Learning applications in telecommunication, autonomous vehicles, cybersecurity, and computer vision. 